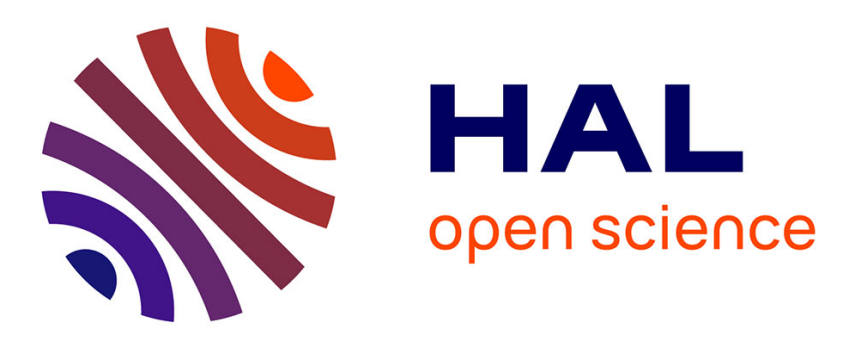

\title{
Wildfire remote sensing with UAVs: A review from the autonomy point of view
}

\author{
Rafael Bailon-Ruiz, Simon Lacroix
}

\section{To cite this version:}

Rafael Bailon-Ruiz, Simon Lacroix. Wildfire remote sensing with UAVs: A review from the autonomy point of view. International Conference on Unmanned Aircraft Systems (ICUAS 2020), Sep 2020, Athens, Greece. hal-02614975

\section{HAL Id: hal-02614975 \\ https://hal.laas.fr/hal-02614975}

Submitted on 21 May 2020

HAL is a multi-disciplinary open access archive for the deposit and dissemination of scientific research documents, whether they are published or not. The documents may come from teaching and research institutions in France or abroad, or from public or private research centers.
L'archive ouverte pluridisciplinaire HAL, est destinée au dépôt et à la diffusion de documents scientifiques de niveau recherche, publiés ou non, émanant des établissements d'enseignement et de recherche français ou étrangers, des laboratoires publics ou privés. 


\title{
Wildfire remote sensing with UAVs: A review from the autonomy point of view
}

\author{
Rafael Bailon-Ruiz ${ }^{1,2}$ and Simon Lacroix ${ }^{1}$
}

\begin{abstract}
This article analyses the state of the art on wildfire remote sensing using UAVs, an application context that has now gained significant interest. It reviews a selection of relevant publications, and proposes a classification scheme to synthesize them from an autonomy perspective. Three metrics are introduced: situation awareness, decisional ability, and collaboration ability. A discussion about the current state and the outlook of UAV systems for wildfire observation concludes the paper.
\end{abstract}

\section{INTRODUCTION}

The most developed application of UAV technology is remote sensing, primarily using image sensors, and more recently LIDARs, in a variety of application contexts: terrain mapping for mining industry, agriculture and flood risk assessment; monitoring and surveillance of long linear structures such as power lines, roads and railway infrastructures; large structure inspection and wildlife monitoring. In the context of forestry, drones have been proved useful for canopy mapping, forest management and wildfire tracking [1].

The consideration of UAVs for wildfire remote sensing has risen in the recent years. A search query in the Web of Science database including publications with contents related to UAVs and wildfires returns 308 records for the 1990 to 2018 range. The distribution of these documents by year, shown in Figure 2, shows an accelerating growth since 2010 in the number of publications, with the total accumulated publication count increasing exponentially as a consequence. As observed by D.J. De Solla Price [2], father of modern scientometrics, this is a clear symptom of an emerging research topic.

To our knowledge, only two surveys about wildfire remote sensing systems using UAVs have been published: [3] and [4]. [3] focus on the analysis of the sensing hardware and algorithms, including a detailed classification of image sensor characteristics and fire detection algorithms. [4] is an overview of the applicability of UAV technology into fire management operations in which the authors also provide a clear synthesis of the institutional and legal state of the art in the USA. Because system architecture and operational autonomy of the vehicles are secondary concerns in both reviews, we deem interesting to discuss the usage of UAVs in wildfire remote sensing from a robotic point of view: studying the interactions between vehicles, control operators and the environment. In other words, how systems are designed to autonomously act with little or without direct human involvement to gather information on wildfires.

\footnotetext{
${ }^{1}$ LAAS-CNRS, Université de Toulouse, CNRS, Toulouse, France

${ }^{2}$ LAAS-CNRS, Université de Toulouse, INSA, Toulouse, France
}

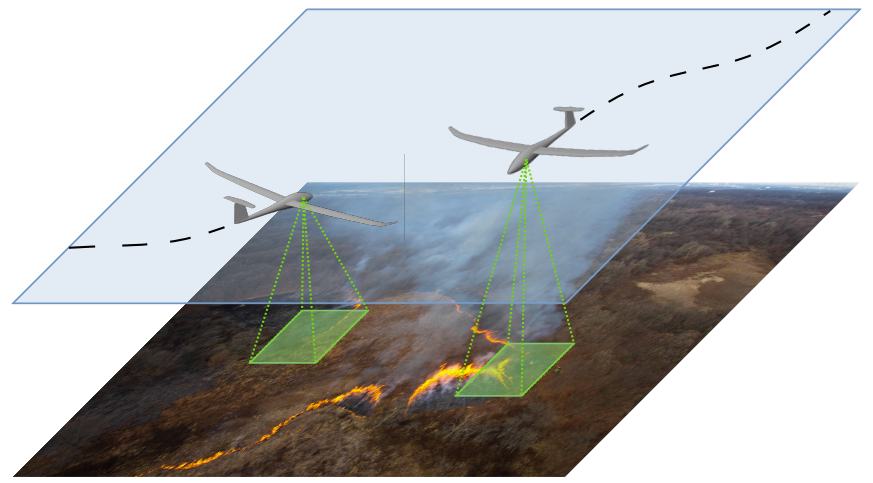

Fig. 1: Low altitude UAVs are excellent means to provide high resolution and timely information on wildfires.

\section{Wildfires and UAV missions}

As reviewed by [4], the expansion of UAV applications in the fire domain area mainly pertains to the domain of wildfire remote sensing. Manned aerial wildfire monitoring is costly and very risky, specially when dealing with uncontrolled fires. Hence, transferring the aircraft operator from the air to the ground improves the cost-effectiveness and efficiency of wildfire fighting efforts [5], freeing resources for other firefighting-related duties. Other tasks beyond remote sensing can be achieved by UAVs, like the aerial ignition of prescribed fires [6], or even firefighting [7], the latter being yet to be developed in operational contexts, as it requires carrying huge quantities of water and fire retardant.

The most common mission in the wildfire remote sensing domain is fire mapping, which produces a map of an area highlighting the locations on fire at a particular time from geo-referenced aerial imagery. Additionally, it is possible to process the fire maps in order to determine the current fire perimeter and to provide an estimation of its position in unobserved areas. When mapping is performed in a continuous manner, for instance to track a fire perimeter to provide regular updates of the fire map, it is referred to as monitoring.

UAVs are able to generate rich and precise fire data with high resolution cameras, that can be used to characterize the fire geometry. Remote 3D reconstruction of a wildfire can give a lot of information to the firefighters, who can safely assess the fire severity in a particular location. Besides, collected data help researchers to better understand fire propagation. The work of [8] and [9] are examples of 3D flame reconstruction algorithms from aerial stereo footage. Aerial thermal infrared imaging can also be used in 


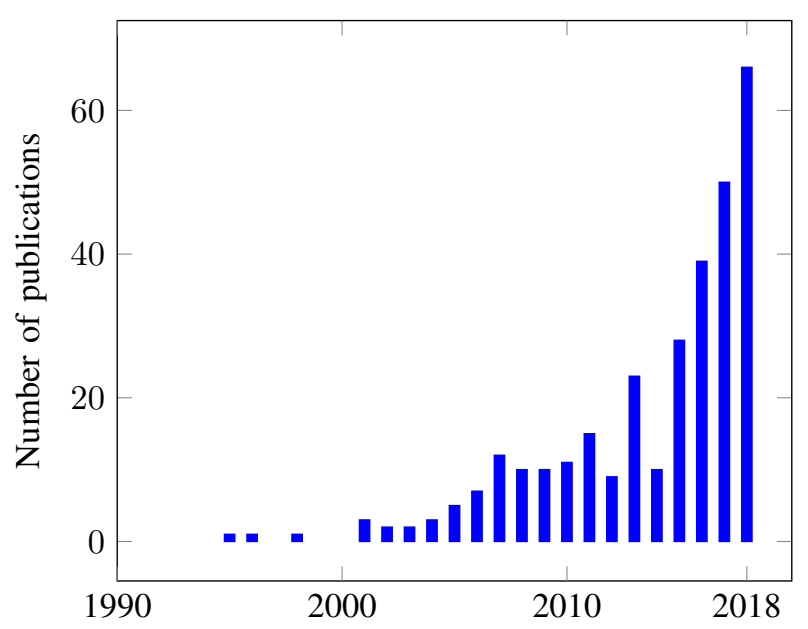

Fig. 2: Number of $U A V$ and wildfire related publications by year from 1990 to 2018.

Obtained using the search query $T S=(($ fire $O R$ wildland $N E A R / 1$ fire $O R$ "forest fire" OR wildfire $\$)$ AND ((UAV\$ OR drone \$ OR "unmanned aerial" OR "unmanned aircraft" OR RPAS OR "uninhabited aerial" OR "uninhabited aircraft") NOT(ant\$OR bee\$OR workerbee\$ OR apis-mellifera OR HIV OR gun \$ OR weapon\$ OR "battle field" OR gunfire\$))) in the Web of Science database.

automated wildfire monitoring. In [10], the proposed system is able to track wildfire perimeters from images acquired by a UAV and use this information to improve the parameters of a wildfire propagation simulator. Such wildfire prognosis capability could be integrated in an automated fire decision support tool, but means of this kind still have some way to go before their use in real operational situations.

\section{Outline}

The next section summarizes and comments the most relevant publications related to wildfire observation systems using UAV technology. Publications have been selected for the novelty of their approach and the relative importance of the citation count, and subsequently put into two distinct categories: systems consisting on a Single $U A V$, and systems built upon Multiple UAVs. This categorization reflects a trend in the state of the art, with systems based on fleets of UAVs becoming more common as UAV technology becomes more powerful. Section III introduces a classification scheme of UAV-based wildfire remote sensing systems in terms of autonomy levels, and synthesizes the state of the art with respect to this taxonomy. A discussion concludes the paper, highlighting the current research trends that we believe relevant for UAVs in the context of wildfires.

\section{RELEVANT PUBLICATIONS AbOUt UAVS AND WILDFIRES}

\section{A. Single UAV systems}

Preliminary work on unmanned aircraft technologies for wildfire remote sensing started in the early 2000's. This period is characterized by the usage of remotely piloted High Altitude and High Endurance UAVs (HALE UAVs) exploited by research agencies as a complement to existing satellite monitoring systems. HALE UAVs feature great capabilities:

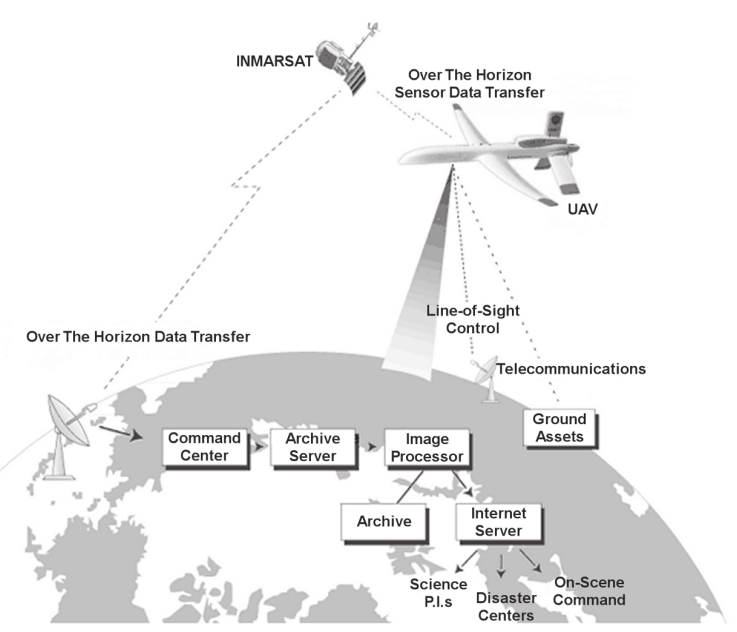

Fig. 3: The FiRE project system architecture. A UAV captures thermal images of an ongoing fire and sends them to a command center through satellite communication. Images are geo-rectified as they arrive, then delivered to hazard response teams. Excerpt from [11]

they can fly for hours at high altitude and carry significantly heavy payloads, buy they are expensive systems and do not provide more precise data than satellites do. Small UAVs fly much closer to the ground potentially being able to sense more detailed information about wildfires.

The objective of the FiRE project [11] is to demonstrate the use of a remotely operated UAV equipped with a thermal scanner for wildfire mapping. Images are transmitted to the ground station via a satellite link and are then georectified to produce a fire map in real time. The system was tested in a controlled burn site in 2001 using an ALTUS II UAV, producing 5 geo-rectified images with a $2.5 \mathrm{~m}$ spatial resolution during a one hour flight. The architecture of the FiRE system, depicted in Figure 3, is probably the first instance of a complete wildfire monitoring system, serving as the foundation of more capable ones to come in the following years.

People from the same laboratory reported in [12] about new fire imaging missions flown by NASA and the US Forest Service with a HALE UAV between 2006 and 2010. This time, the UAV included a hyperspectral camera and sufficient computing power to process sensor data on-board. A fire hotspot algorithm running on the UAV on-board CPU was able to detect burning areas by applying a threshold to selected infrared bands. With the help of a digital elevation model, image regions corresponding to fire were projected over the ground level to obtain a geo-rectified fire map. Finally, the fire map was sent in real time to a Wildfire Collaborative Decision Environment that integrated multiple geospatial sources allowing real-time collaborative manipulation of the information.

OSIRIS [13] is a European project whose objective is to develop a High Altitude Platform for wildfire monitoring using a solar-powered HALE UAV. This UAV is able to 
follow a predefined flight plan that can be updated at any time from a Ground Control Station. While achieving the mission, the aircraft is able to capture high-resolution images that are transmitted in real time to the ground station through a satellite link. Then, the raw images can be forwarded to a Central Data Processing Center for additional processing, that archives all the geo-referenced imagery that has been produced and allows the final users to consult the information. No automated assessment is performed on the images to detect fire spots or propagation perimeters. We could not assess whether this work has been validated in realistic conditions or not.

[14] describes the results of a project devoted to wildfire monitoring with a remote sensing system based on two platforms: a small UAV and a two-seat airplane. Different sensing instruments were available including thermal, multispectral and hyperspectral cameras.

The work of [15] assesses the interest of using UAVs to observe wildfires from the point of view of a firefighting team. Once a fire is declared, the first task of the response crew is the reconnaissance of the situation. Given that the damage generated by a wildfire depends greatly on the response time, the earlier firemen have an understanding on the severity of the fire, the earliest they can apply the best countermeasures. Hence, air reconnaissance with UAVs being operated directly by the fire fighting crew seems for the author the most effective way to get early information about a starting wildfire. When the fighting efforts are concentrated on a specific area, UAVs can be used as a decision support tool for the least active portions of the front. After doing a simple fire reconnaissance test with a regular commercial $\mathrm{UAV}$, the author concludes with a roadmap for three wildfire monitoring designs that firefighting crews would benefit from. Its last milestone consists of fleets of autonomous UAVs including blimps, helicopters and fixed-wing aircraft.

An autonomous wildfire monitoring system is depicted in [16], whose objective is to track a set of hot-spots as fast as possible. Given a realistic simulation of the expected evolution of a wildfire, a hot-spot is defined by the center of a cluster of locations where the fire is the fastest growing. The authors introduce a greedy algorithm that guides the UAV towards these hot-spots. Then, the algorithm is evaluated against a baseline strategy consisting in circling the current fire contour. A mixed-reality experiment, with a real UAV and a simulated fire has been performed to test the proposed algorithm.

\section{B. Multiple UAV systems}

The use of multiple UAVs reveals new forms of operation. The scale of a wildfire is often too large to be encompassed by a single UAV, which fleets of UAVs can cope with. But systems built upon multiple UAVs also imply new design challenges, as vehicles must communicate and collaborate in some way to exploit the full potential of the fleet. Hence, some task allocation or distributed control algorithms are necessary to operate the fleet.

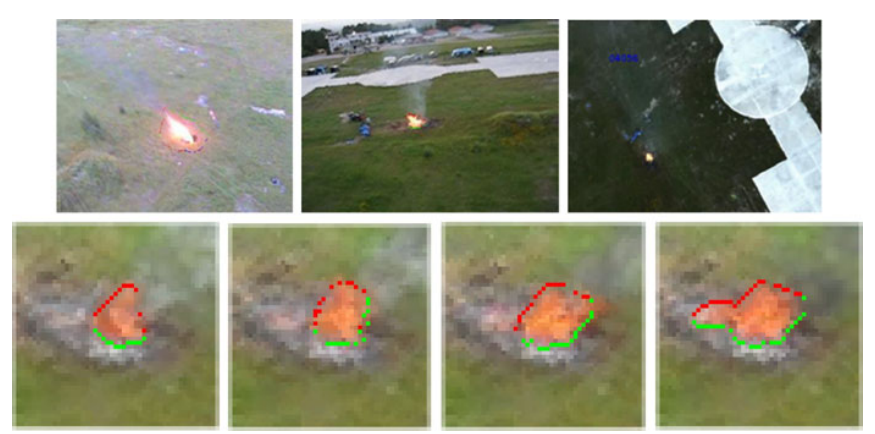

Fig. 4: Aerial view of a wildfire from different angles (top). Algorithms are able to locate the position of the fire if a digital elevation map is provided (bottom). Excerpt from [20]

The interest in systems based on multiple UAVs is supported by a recent publication [17] that evaluates different wildfire remote sensing schemes, combining land sensors, fixed-wing and rotary-wing UAVs, and satellites. The main interest of this work is the analysis of different sensing strategies regarding how they fit into the fulfillment of typical missions, i.e. fast and reliable wildfire detection and robust monitoring. The authors retain three unique strategies: the first one using satellite and land sensor imagery, the second one built upon fleets of drones and the third one combining fleets of UAVs with ground cameras. UAV-based systems are found to be the most useful configuration overall with respect to their criteria.

To the extent of our knowledge, the first publication devising the application of fleets of fixed-wing UAVs as a wildfire remote sensing platform is [18], in which the authors conceive a remote sensing architecture using UAVs as a foreseeable alternative to satellites. The fleet, flying at stratospheric level, carries optical payloads and has on-board processing capabilities. The work presented in this article is more centered into the design of the optical payload, but the introduction of the idea of a UAV fleet platform with onboard processing for autonomous detection and tracking of ground phenomena was innovative at that time.

The authors of [19] propose a fire spot detection system in the context of the COMETS project. This system included for the first time a fleet of heterogeneous UAVs composed of three vehicles of two different kinds and with varying levels of autonomy: autonomous and remote controlled helicopters, and a remote piloted blimp. The fire spotting algorithm relies on the collaboration between UAVs featuring IR cameras and other UAVs carrying visible cameras to increase the detection probability. Once a fire spot is confirmed, the assessment is completed by observing it from different points of view. This system has been tested on the field and authors provide an extensive report on the results of the experiment. More recent publications from the same authors introduce improved results on fire contour extraction [20] and 3D fire shape estimation from multiple ground and aerial views [8].

Other innovative work using fleets of UAVs is introduced in [21]. This publication depicts a collaborative wildfire monitoring system using a fleet of LASE (Low Altitude Short 
Endurance) fixed-wing UAVs. The monitoring framework relies on a decentralized algorithm that makes each UAV track the front of a round shape wildfire to measure the total length of the perimeter. The UAVs fly in opposite directions and every time a vehicle meets another one, they exchange information about the length of the perimeter they have already tracked. Given sufficient time, the fleet agrees on the front length, equally distributing the portion of the front being monitored by every vehicle. The interest of the proposed system resides in its ability to work in limited communication scenarios, with information being sent from one agent to another, reaching at the end the ground station. This time, the algorithm was tested with the help of a wildfire simulator and a 6 degree of freedom simulator for the UAVs.

[22] describes a monitoring architecture to estimate the perimeter of a wildfire using a fleet of UAVs. Their model defines the fire front as a set of discrete control points that move outwards the center of the fire. Then, the border is observed by a fleet of UAVs equipped with a binary sensor capable of detecting whether a UAV is located over the wildfire or not. Observations are fed into a tailored Kalman filter that estimates the location of the perimeter control points, and associates to each of them an uncertainty value. A planning algorithm creates a plan for fleets of UAVs to observe the control points prioritizing those whose location is more uncertain.

[23] proposes an early fire detection platform with ground sensors and a fleet of UAVs. The suggested scheme advocates for the use of small quad-rotor drones for early fire verification and a blimp equipped with a smoke detector and a radiometer for hot-spot monitoring.

FireRS [24] is a European project to which we participated, that implements a wildfire detection and mapping system to support the decision-making procedures of emergency response teams. A network of land-based fire sensors, distributed across the wild land, scans the area for fire ignitions and estimates their location. When a wildfire is detected, an alarm is transmitted by the means of satellite communication, to an autonomous control center that deploys a fleet of small UAVs for continuous wildfire detection and mapping. The control center runs an observation planning algorithm tailoring a local search approach to plan trajectories for the UAVs exploiting realistic models of the environment, the wildfire and of the UAVs [25] [26].

A novel swarm control algorithm for wildfire search and tracking is proposed in [27]. This algorithm for fleets of microUAVs is based on a dynamic pheromone map, which combined with a particular heuristic, makes the swarm search for new fire spots while tracking existing ones in a distributed manner. Each UAV carries an infrared camera capable of detecting and geo-locating a fire. Periodically, pheromone maps are shared between agents with the objective of collaboratively building a complete fire map. This wildfire tracking algorithm has been tested in simulation over a synthetic ovalshaped fire.

[28] proposes a distributed control framework for a fleet of rotary-wing UAVs that spatially distributes the vehicles

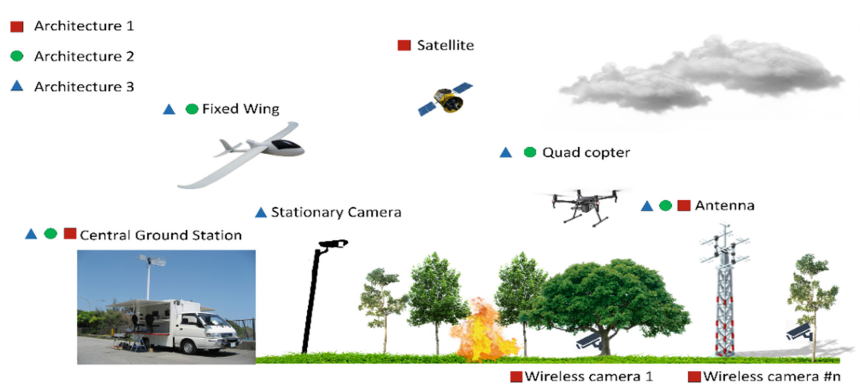

Fig. 5: Due to the extension and the duration of wildfires, teams of vehicles, multiple sensors and communication schemes must be considered for a successful wildfire monitoring mission. Excerpt from [17]

to observe a wildfire. The algorithm tries to maximize the fire coverage, moving the vehicles to the most advantageous positions while the fire keeps spreading.

[7] applies a deep reinforcement learning strategy for a fleet of fire fighting UAVs. The purpose of the proposed algorithm is to control the propagation of a wildfire by commanding the UAVs to, first, detect trees close to ignition and second, drop some retardant to stop the fire spread.

A wildfire rate of spread estimation algorithm using data obtained by a fleet of rotary-wing UAVs is presented in [29], and further developed in [30] and [31]. The estimation exploits a Kalman filter that combines past knowledge about the fire perimeter with measurements to predict the current contour position. Thanks to a wildfire simulator, the authors tested the algorithm inside a realistic environment.

[32] introduces an autonomous wildfire surveillance system, using a deep reinforcement learning approach to control a fleet of UAVs. The problem is modeled after a Partially Observable Markov Decision Process for the UAV controller state and actions, and a stochastic wildfire model is used to generate the training environment. Two different strategies for generating actions for each UAV in the fleet are depicted. The first one directly derives actions from binarised images of the wildfire around each UAV (burning or not burning). A penalty system favors the aircraft to follow the fire front while avoiding extreme bank angles and flying close to other UAVs. The second strategy defines an ignition belief map for the whole fire, encoding for every cell whether it is on fire and the time elapsed since the last observation. Each time an UAV flies over an area, the belief map is updated and the fleet obtains a reward when a cell thought to be extinguished is seen on fire. Deep reinforcement learning is used to train the controllers for the two aforementioned strategies. The authors study the pros and cons of each approach and analyze their performance against each other under several distinct scenarios. The results show that both approaches are able to track wildfires, none of them outperforming the other in any case.

\section{AUtONOMY LEVEL CLASSIFICATION SCHEME}

This section synthesizes the publications with respect to the Autonomy of the systems, defined as the ability of a robot 
to perform a given task with the least human involvement.

Several pieces of work have dealt with the issue of classifying unmanned aerial systems by their Autonomy abilities. A study by the US Air Force Research Laboratory [33] proposes an Autonomous Control Level (ACL) metric divided in 10 levels, from Remotely piloted to Fully Autonomous Swarms, based on three metrics: Perception/Situational Awareness; Analysis/Decision Making; and Communication/Cooperation. The Autonomy Levels for Unmanned Systems (ALFUS) [34] is a popular classification by the USA National Institute of Standards and Technology that takes in account three aspects of the overall completion of a mission: mission complexity, environmental complexity, and human independence. A working group of NATO has classified UAS autonomy in 4 levels [35]: remotely controlled system, automated system, autonomous non-learning system, and autonomous learning system.

These three classification schemes have been established considering mostly military Unmanned Aerial Systems, and some of their definitions are not suitable to fully describe civil missions. The scales for the different autonomy levels depend on specific abilities such as threat detection and flight tactics that are not fully compatible with wildfire remote sensing particularities. Nevertheless, the principles that define these classifications can be adapted to the remote sensing context.

\section{A. Main metrics}

We propose the following three following metrics to establish a classification of UAV based wildfire monitoring systems:

Awareness Whether the system is able to provide a global understanding and analysis of the fire state and properties.

Decision Whether the system is able to decide which actions to perform and achieve them.

Collaboration Whether the system components communicate, share information or perform joint tasks.

An important difference with respect to previous work is the enlargement of the analysis scope to the overall system. The classification is no longer restricted to the elements that are embedded into the UAVs but considers all the system devices, protocols and algorithms that constitute the whole system: satellites, ground sensors, ground control station, etc.

The autonomy level of a system is described by these three main metrics, seen as mostly independent qualitative dimensions. The proposed classification does not provide numerical scores, but a qualitative synthesis of system strengths.

1) Awareness: The Awareness metric extends elementary fire perception abilities (e.g. fire detection) to the analysis of the observed data. It represents the ability to synthesize observations into a computerized understanding of the observed phenomenon.

The Awareness metric is divided into three levels:

No data analysis Acquired data is disseminated with no further analysis.
Feature extraction The system is able to detect some features of a wildfire from sensor data - for example, detecting fire hot-spots.

Situation assessment The system is able to exploit sensor data and models to provide additional insights about the wildfire, such as estimation of missing data or prediction of wildfire evolution.

2) Decision: The Decision metric defines level of intervention of the users on the definition of the actions to be carried out by an UAV.

This metric presents two distinct stages of increasing levels of autonomy, depending on whether the UAV adapts its actions with respect to the environmental or not. At the first stage, UAVs are able to navigate by themselves following a human-made plan specifying how to perform the mission. For instance: "Take off, then go over point A, take a picture, and land at point B". At the second stage, the mission plan is not fully specified by an operator: it is automatically generated from high-level requirements and constraints provided by the users. For example: "Find fires in this region" or "monitor this fire perimeter".

These two stages are detailed in the following four autonomy levels of the decision metric:

Remotely piloted The UAV is directly piloted by an operator.

Manual planning The UAV is able to navigate autonomously given a plan provided by a human operator.

Autonomous planning The UAV is able to navigate autonomously a trajectory generated by a computer taking in account the current or expected environment.

Adaptive autonomous planning The UAV is able to navigate autonomously a planned trajectory and modify it while flying according to changes in the environment.

3) Collaboration: The Collaboration metric evaluates the existence and the autonomy level of interaction between several UAVs. Given the spatial and time extents of wildfires, the benefits of multiple vehicles for observation missions are clear. Nevertheless, having a fleet of vehicles inevitably requires taking in account a collaboration dimension in the design, as the control algorithms must be adapted to this configuration. In this respect, two classes of designs can be found: one approach is to have a fleet of UAVs with independently allocated tasks executing actions in parallel to achieve the mission. For instance, the monitoring of a $10 \mathrm{~km}^{2}$ region can be done with 10 UAVs patrolling areas of $1 \mathrm{~km}^{2}$. Another design approach is to make UAVs work together, exploiting synergies to achieve a common objective. Good examples of this strategy are [21] and [19]. The first is an instance of swarm design, that yields the estimation of a fire perimeter length with a fleet of vehicles having limited sensing thanks to local information exchange. In the second case, UAVs carrying different sensors observe the same fire from different locations to increase the accuracy of the detection. 
This metric is divided into the following three levels:

One vehicle No collaboration.

Distributed task Multiple UAVs achieve independently allocated tasks.

Cooperative task Multiple UAVs for which one vehicle task execution requires the interaction with one or more other vehicles.

\section{B. Secondary concerns}

There are other aspects playing a complementary role into the characterization of wildfire remote sensing systems. These concerns do not take part directly of the autonomy definition, but are still worth to be mentioned as they help put those designs into context.

1) On-board versus off-board data processing: On-board processing means that the vehicles are capable to process sensor data and to produce a synthesis of the information on their own. On the contrary, off-board processing defines a vehicle that only acts as the carrier of a sensor, transmitting raw data or storing it for further retrieval. The distinction between on-board and off-board computation is not sharp, as data processing efforts may be shared between the vehicles and the ground station.

The choice of a data processing configuration comes out as a technical consequence of multiple combined factors: 1) The ratio between the performance of the communication link and the size of the data that needs to be transmitted, 2) The computing power that can be embedded into the UAVs, and 3) Mission-specific requirements.

2) UAV airframe type: UAVs may be classified by their structure and method of lift, some airframes being more suitable for specific types of mission than others.

Lighter-than-air vehicles are able to fly during long periods of time and can control their trajectories, but they navigate very slowly and are prone to winds. This kind of vehicle is suitable for static surveillance missions as used in [20], taking pictures from a high point, and long term observation of extinguished areas like in [23], but not for wildfire perimeter tracking when wind is one of the main cause of the fire propagation.

Rotary-wing aircraft can take off and land vertically and hover, but they require the continuous action of the rotors to fly, reducing their endurance. Quadrotors, and multirotor aircraft in general, have recently gained some popularity thanks to the introduction of relatively cheap commercial off-the-shelf models.

In contrast, fixed-wing planes have to be continuously moving forward, they are not able to hover and are nonholonomic. Nevertheless, they are very useful as their airspeed and endurance are high. These two reasons make them the airframe of choice for fire mapping and tracking missions.

New UAV designs with hybrid airframes are capable of vertical take-off and landing, and horizontal flying, combining the advantages of rotary-wing and fixed-wing aircraft designs, but are not yet widespread.
3) Mission type: In the context of wildfires, UAV missions are devoted to information gathering, be it detection, mapping, monitoring or tracking of either hot-spots or fire perimeters (also referred to as fire front). Fire suppression, one of the objectives of [7], is the sole mission that does not fall into the remote sensing category.

Detection missions consist in finding the existence of fire and its coordinates. The objective of a mapping mission, typically performed during the early stages of a wildfire, is to build a map of the fire extent. Depending on the kind of sensor used, visible or thermal, this can be for the fire front or hot-spots. When mapping is done continuously for a region as a whole, the mission type is monitoring; or tracking, if a particular feature of the wildfire is being followed. Surveillance is a variant of the monitoring mission type where UAVs are used to observe an area before or after a fire is declared.

4) Field tests: The maturity of research and development can be assessed on whether it has been tested in real conditions or not. Among all the systems reviewed, those that have been field tested have single vehicle configurations (with [19] as the sole exception). This fact is consistent with the analysis of [1] relating current legal issues of UAV usage. It can be expected that in the coming years there is going to be a push for the authorization of multiple UAV operations, as technology gets more mature.

\section{Discussion}

Table I synthesizes the work reviewed in section II with respect to the metrics introduced in section III. Publications are sorted by year and grouped into one record when they describe parts of the same system. Additionally, Figure 6 shows a condensed pairwise comparison of the most common wildfire remote monitoring system configurations with respect to the defined autonomy level metrics. The charts highlight the most frequent levels and those that are not popular.

As expected, we observe an overall increasing level in autonomy in the recent years. In particular, every publication after [20] in 2012 describes a system featuring some kind of Situation Assessment, that is either creating some sort of map of the wildfire location, or recovering the fire geometry: Situation Assessment is naturally the first step to autonomous wildfire monitoring. Furthermore, most of recent work in the literature reach the autonomous planning decision level with multiple UAVs. This is a consequence of the nature of wildfires, which extend both in space and time, and of the requirements of the operators, who need information as timely and as complete as possible. As a result, it is likely that fleets of autonomous UAVs are going to be the standard for future wildfire remote sensing developments.

If the recent trend in publications shows that using multiple UAVs are possibly the best fit to observe wildfires, such technology is not completely ready yet. There is a general lack of extensive field experiments that may be due to a couple of overcoming challenges. The first is legal: flight restrictions require a dedicated safety pilot for every 
vehicle, which is an issue for fleets of autonomous UAVs. The second may be due to autonomy challenges: reaching the collaboration level of autonomy requires an advanced level of decisional autonomy. As illustrated in Figure 6c, no system with multiple UAVs is remotely piloted.

Besides, Figure $6 \mathrm{~b}$ illustrates well that autonomous planning systems must rely on situation assessment algorithms. Finally, one can state that the future of wildfire remote sensing is dependent on a general increase in the autonomy of UAV technology. Additional field testing will be required in order to certify collaborative UAV activities, for which system-oriented designs that explicitly account for the ground control station and operator involvement are deemed necessary.

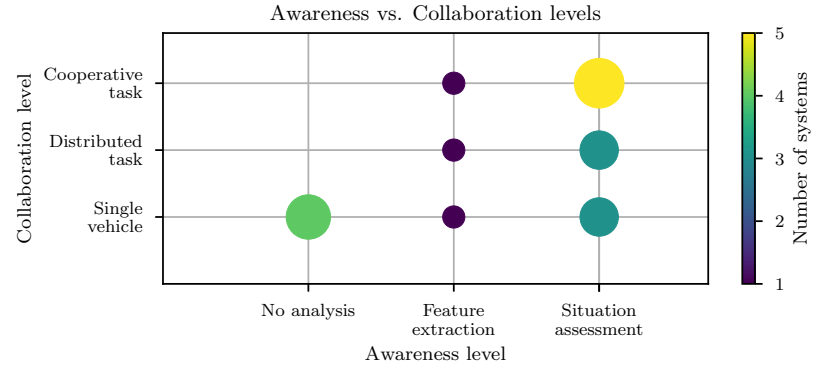

(a) Awareness vs. collaboration autonomy

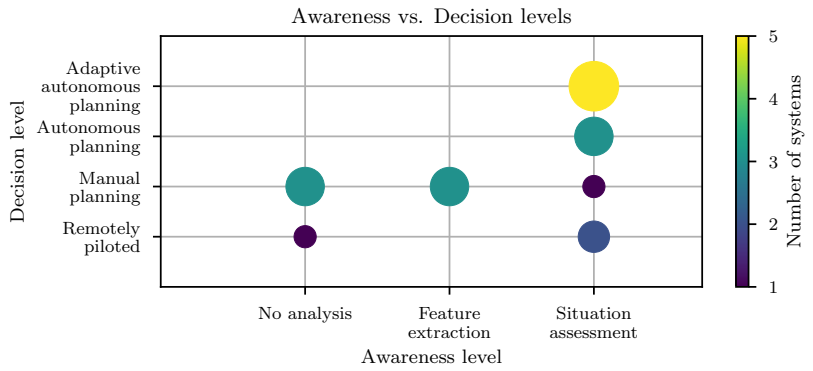

(b) Awareness vs. decision autonomy

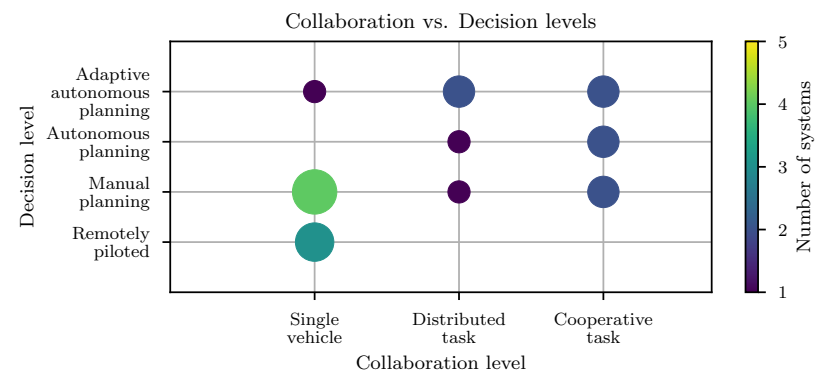

(c) Collaboration vs. decision autonomy

Fig. 6: Examination of the wildfire remote monitoring systems reviewed in this survey. Charts provide aggregated pairwise comparisons of the awareness, decision and collaboration autonomy levels highlighting the most common configurations. The size and the color of the circles indicate the number of contributions. 


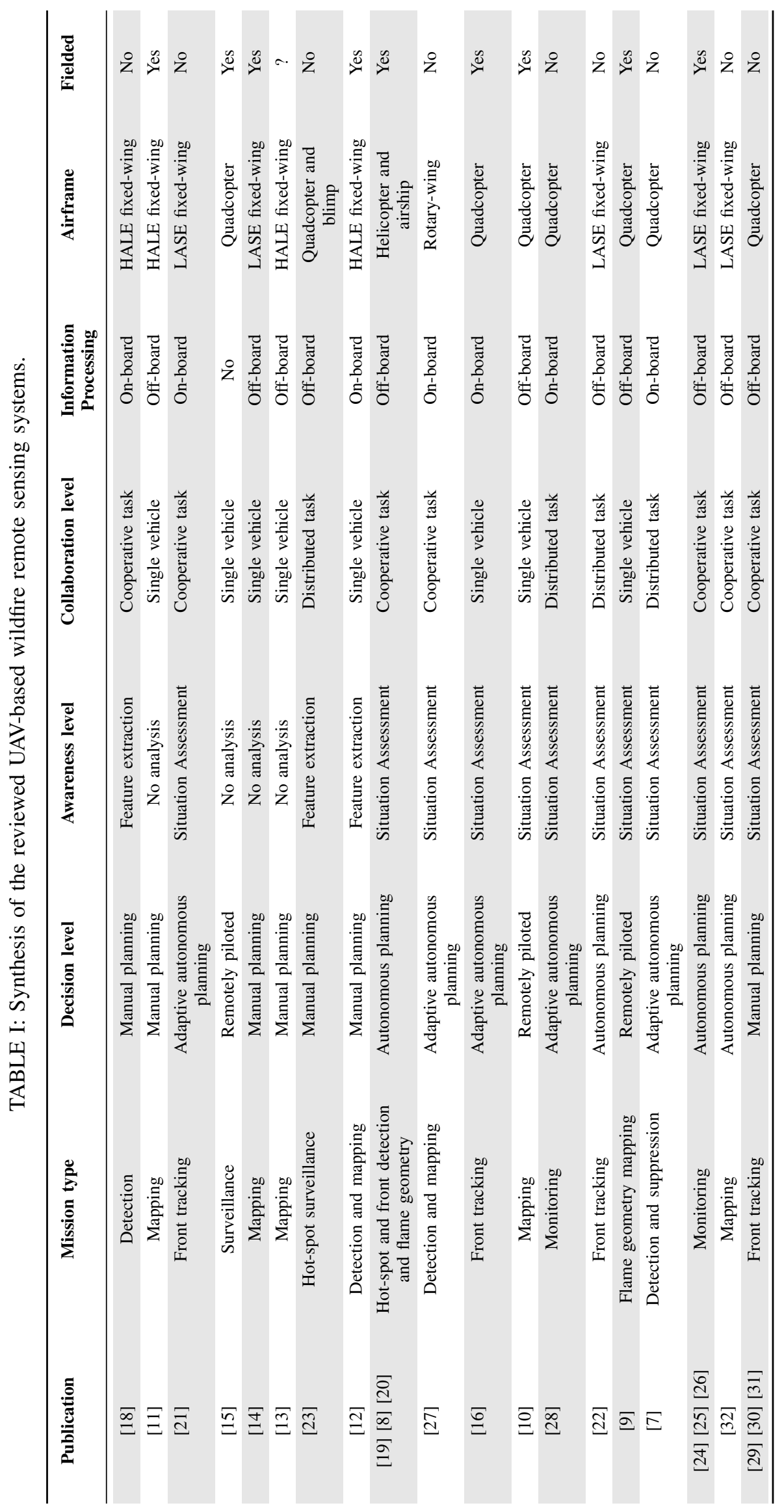




\section{REFERENCES}

[1] L. Tang and G. Shao, "Drone remote sensing for forestry research and practices," Journal of Forestry Research, vol. 26, no. 4, pp. 791-797, Dec. 2015.

[2] D. J. d. S. Price, Little Science, Big Science. Columbia University Press, 1963.

[3] C. Yuan, Y. Zhang, and Z. Liu, "A survey on technologies for automatic forest fire monitoring, detection, and fighting using unmanned aerial vehicles and remote sensing techniques," Canadian Journal of Forest Research, vol. 45, no. 7, pp. 783-792, July 2015

[4] D. Twidwell, C. R. Allen, C. Detweiler, J. Higgins, C. Laney, and S. Elbaum, "Smokey comes of age: Unmanned aerial systems for fire management," Frontiers in Ecology and the Environment, vol. 14, no. 6, pp. 333-339, Aug. 2016.

[5] B. R. Christensen, "Use of UAV or remotely piloted aircraft and forward-looking infrared in forest, rural and wildland fire management: Evaluation using simple economic analysis," New Zealand Journal of Forestry Science, vol. 45, Sept. 2015.

[6] E. Beachly, C. Detweiler, S. Elbaum, B. Duncan, C. Hildebrandt, D. Twidwell, and C. Allen, "Fire-Aware Planning of Aerial Trajectories and Ignitions," in 2018 IEEE/RSJ International Conference on Intelligent Robots and Systems (IROS). Madrid,Spain: IEEE, 2018, pp. 685-692.

[7] R. N. Haksar and M. Schwager, "Distributed Deep Reinforcement Learning for Fighting Forest Fires with a Network of Aerial Robots," in 2018 IEEE/RSJ International Conference on Intelligent Robots and Systems (IROS), ser. IEEE International Conference on Intelligent Robots and Systems. Madrid,Spain: IEEE, 2018, pp. 1067-1074.

[8] J. R. Martínez-de Dios, L. Merino, F. Caballero, and A. Ollero, "Automatic Forest-Fire Measuring Using Ground Stations and Unmanned Aerial Systems," Sensors, vol. 11, no. 6, pp. 6328-6353, June 2011.

[9] V. Ciullo, L. Rossi, T. Toulouse, and A. Pieri, "Fire geometrical characteristics estimation using a visible stereovision system carried by unmanned aerial vehicle," in 2018 15th International Conference on Control, Automation, Robotics and Vision (ICARCV). Singapore: IEEE, 2018, pp. 1216-1221.

[10] M. M. Valero, O. Rios, C. Mata, E. Pastor, and E. Planas, "An integrated approach for tactical monitoring and data-driven spread forecasting of wildfires," Fire Safety Journal, vol. 91, no. Supplement C, pp. 835-844, July 2017.

[11] V. Ambrosia, S. Wegener, D. Sullivan, S. Buechel, S. Dunagan, J. Brass, J. Stoneburner, and S. Schoenung, "Demonstrating UAVacquired real-time thermal data over fires," Photogrammetric Engineering \& Remote Sensing, vol. 69, no. 4, pp. 391-402, Apr. 2003.

[12] V. G. Ambrosia, S. Wegener, T. Zajkowski, D. V. Sullivan, S. Buechel, F. Enomoto, B. Lobitz, S. Johan, J. Brass, and E. Hinkley, "The Ikhana unmanned airborne system (UAS) western states fire imaging missions: From concept to reality (2006-2010)," Geocarto International, vol. 26, no. 2, SI, pp. 85-101, 2011.

[13] N. Lewyckyj, J. Biesemans, and J. Everaerts, "OSIRIS: A European project using a High Altitude Platform for forest fire monitoring," in Safety and Security Engineering II, ser. WIT Transactions On The Built Environment, Guarascio, M and Brebbia, CA and Garzia, F, Ed., vol. 94. Southampton, England: WIT Press/Computational Mechanics Publications, 2007, pp. 205-213.

[14] F. Esposito, G. Rufino, A. Moccia, P. Donnarumma, M. Esposito, and V. Magliulo, "An integrated electro-optical payload system for forest fires monitoring from airborne platform," in 2007 IEEE Aerospace Conference. Big Sky, Montana: IEEE, 2007, pp. 2015-2027.

[15] A. Restas, "Forest fire management supporting by UAV based air reconnaissance results of Szendro Fire Department, Hungary," in First International Symposium on Environment Identities and Mediterranean Area. Corte-Ajaccio, France: IEEE, 2006, pp. 84-88.

[16] R. C. Skeele and G. A. Hollinger, "Aerial Vehicle Path Planning for Monitoring Wildfire Frontiers," in Field and Service Robotics: Results of the 10th International Conference, ser. Springer Tracts in Advanced Robotics, Wettergreen, DS and Barfoot, TD, Ed., vol. 113, Toronto, Canada, 2016, pp. 455-467.

[17] V. C. Moulianitis, G. Thanellas, N. Xanthopoulos, and N. A. Aspragathos, "Evaluation of UAV Based Schemes for Forest Fire Monitoring," in Advances in Service and Industrial Robotics, ser. Mechanisms and Machine Science, N. A. Aspragathos, P. N. Koustoumpardis, and V. C. Moulianitis, Eds. Cham: Springer International Publishing, 2019, pp. 143-150.
[18] F. Dovis, L. Lo Presti, E. Magli, P. Mulassano, and G. Olmo, "Stratospheric platforms: A novel technological support for earth observation and remote sensing applications," in Sensors, Systems and Next-Generation Satellites $V$, ser. Proceedings of SPIE, Fujisada, H and Lurie, JB and Weber, K, Ed., vol. 4540. Bellingham, WA, USA: Spie-Int Soc Optical Engineering, 2001, pp. 402-411.

[19] L. Merino, F. Caballero, J. R. Martínez-de Dios, and A. Ollero, "Cooperative fire detection using unmanned aerial vehicles," in Proceedings of the 2005 IEEE International Conference on Robotics and Automation. Barcelona, Spain: IEEE, 2005, pp. 1884-1889.

[20] L. Merino, F. Caballero, J. R. Martínez-de-Dios, I. Maza, and A. Ollero, "An Unmanned Aircraft System for Automatic Forest Fire Monitoring and Measurement," Journal of Intelligent \& Robotic Systems, vol. 65, no. 1, pp. 533-548, Jan. 2012.

[21] D. W. Casbeer, D. B. Kingston, R. W. Beard, and T. W. McLain, "Cooperative forest fire surveillance using a team of small unmanned air vehicles," International Journal of Systems Science, vol. 37, no. 6, pp. 351-360, May 2006.

[22] S. Rabinovich, R. E. Curry, and G. H. Elkaim, "Toward Dynamic Monitoring and Suppressing Uncertainty in Wildfire by Multiple Unmanned Air Vehicle System," Journal of Robotics, 2018.

[23] N. von Wahl, S. Heinen, H. Essen, W. Kruell, R. Tobera, and I. Willms, "An integrated approach for early forest fire detection and verification using optical smoke, gas and microwave sensors," in Modelling, Monitoring and Management of Forest Fires II, ser. WIT Transactions on Ecology and the Environment, Perona, G and Brebbia, CA, Ed., vol. 137. Southampton, England: WIT PRESS, 2010, pp. 97+.

[24] F. Pérez-Lissi, F. Aguado-Agelet, A. Vázquez, P. Yañez, P. Izquierdo, S. Lacroix, R. Bailon-Ruiz, J. Tasso, A. Guerra, and M. Costa, "FIRERS: Integrating land sensors, cubesat communications, unmanned aerial vehicles and a situation assessment software for wildland fire characterization and mapping," in 69th International Astronautical Congress, Bremen, Germany, Oct. 2018

[25] A. Bit-Monnot, R. Bailon-Ruiz, and S. Lacroix, "A local search approach to observation planning with multiple uavs," in Twenty-Eighth International Conference on Automated Planning and Scheduling, 2018.

[26] R. Bailon-Ruiz, A. Bit-Monnot, and S. Lacroix, "Planning to Monitor Wildfires with a Fleet of UAVs," in 2018 IEEE/RSJ International Conference on Intelligent Robots and Systems (IROS). Madrid, Spain: IEEE, 2018, pp. 4729-4734.

[27] D. J. Howden, "Fire Tracking with Collective Intelligence using Dynamic Priority Maps," in 2013 IEEE Congress on Evolutionary Computation (CEC). Cancun, Mexico: IEEE, 2013, pp. 2610-2617.

[28] H. X. Pham, H. M. La, D. Feil-Seifer, and M. Deans, "A Distributed Control Framework for a Team of Unmanned Aerial Vehicles for Dynamic Wildfire Tracking," in 2017 IEEE/RSJ International Conference on Intelligent Robots and Systems (IROS), Bicchi, A and Okamura, A, Ed. Vancouver, Canada: IEEE, 2017, pp. 6648-6653.

[29] Z. Lin and H. H. T. Liu, "Enhanced Cooperative Filter for Wildfire Monitoring," in 54th IEEE Conference on Decision and Control (CDC). Osaka, Japan: IEEE, 2015, pp. 3075-3080.

[30] _ , "Topology-based distributed optimization for multi-UAV cooperative wildfire monitoring," Optimal Control Applications and Methods, vol. 39, no. 4, pp. 1530-1548, 2018.

[31] Z. Lin, H. H. T. Liu, and M. Wotton, "Kalman Filter-Based LargeScale Wildfire Monitoring With a System of UAVs," IEEE Transactions on Industrial Electronics, vol. 66, no. 1, pp. 606-615, Jan. 2019.

[32] K. D. Julian and M. J. Kochenderfer, "Distributed Wildfire Surveillance with Autonomous Aircraft Using Deep Reinforcement Learning," Journal of Guidance Control and Dynamics, vol. 42, no. 8, pp. 1768-1778, Aug. 2019.

[33] B. Clough, "Metrics, Schmetrics! How Do You Track a UAV's Autonomy?" in 1st Technical Conference and Workshop on Unmanned Aerospace Vehicles. Portsmouth, Virginia: American Institute of Aeronautics and Astronautics, May 2002.

[34] H.-M. Huang, "Autonomy Levels for Unmanned Systems (ALFUS) Framework: Safety and Application Issues," in Proceedings of the 2007 Workshop on Performance Metrics for Intelligent Systems, ser. PerMIS '07. Washington, D.C.: ACM, 2007, pp. 48-53.

[35] M. Protti and R. Barzan, "UAV Autonomy-Which level is desirable?which level is acceptable? Alenia Aeronautica Viewpoint," Alenia Aeronautica SpA, Tech. Rep., 2007. 